\title{
Efficient Generation and Processing of Word Co-occurrence Networks Using corpus2graph
}

\author{
Zheng Zhang \\ LIMSI, CNRS, \\ Université Paris-Saclay \\ Orsay, France \\ Ruiqing Yin \\ LIMSI, CNRS, \\ Université Paris-Saclay \\ Orsay, France \\ LRI, Univ. Paris-Sud, CNRS, ruiqing.yin@limsi.fr \\ Université Paris-Saclay \\ Orsay, France \\ zheng.zhang@limsi.fr
}

\author{
Pierre Zweigenbaum \\ LIMSI, CNRS, \\ Université Paris-Saclay \\ Orsay, France \\ pz@limsi.fr
}

\begin{abstract}
Corpus2graph is an open-source NLPapplication-oriented Python package that generates a word co-occurrence network from a large corpus. It not only contains different built-in methods to preprocess words, analyze sentences, extract word pairs and define edge weights, but also supports user-customized functions. By using parallelization techniques, it can generate a large word co-occurrence network of the whole English Wikipedia data within hours. And thanks to its nodes-edges-weight three-level progressive calculation design, rebuilding networks with different configurations is even faster as it does not need to start all over again. This tool also works with other graph libraries such as igraph, NetworkX and graph-tool as a front end providing data to boost network generation speed.
\end{abstract}

\section{Introduction}

Word co-occurrence networks are widely used in graph-based natural language processing methods and applications, such as keyword extraction (Mihalcea and Tarau, 2004) and word sense discrimination (Ferret, 2004).

A word co-occurrence network is a graph of word interactions representing the co-occurrence of words in a corpus. An edge can be created when two words co-occur within a sentence; these words are possibly non-adjacent, with a maximum distance (in number of words, see Section 2.2) defined by a parameter $d_{\max }$ (Cancho and Solé, 2001). In an alternate definition, an edge can be created when two words co-occur in a fixed-sized sliding window moving along the entire document or sentences (Rousseau and Vazirgiannis, 2013). Despite different methods of forming edges, the structure of the network for sentences will be the same for the two above definitions if the maximum distance of the former is equal to the sliding window size of the latter. Edges can be weighted or not. An edge's weight indicates the strength of the connection between two words, which is often related to their number of co-occurrences and/or their distance in the text. Edges can be directed or undirected (Mihalcea and Radev, 2011).

While there already exist network analysis packages such as NetworkX (Hagberg et al., 2008), igraph (Csardi and Nepusz, 2006) and graph-tool (Peixoto, 2014), they do not include components to make them applicable to texts directly: users have to provide their own word preprocessor, sentence analyzer, weight function. Moreover, for certain graph-based NLP applications, it is not straightforward to find the best network configurations, e.g. the maximum distance between words. A huge number of experiments with different network configurations is inevitable, typically rebuilding the network from scratch for each new configuration. It is easy to build a word co-occurrence network from texts by using tools like textexture ${ }^{1}$ or GoWvis ${ }^{2}$. But they mainly focus on network visualization and cannot handle large corpora such as the English Wikipedia.

Our contributions: To address these inconveniences of generating a word co-occurrence network from a large corpus for NLP applications, we propose corpus2graph, an open-source ${ }^{3}$ NLP-oriented Python package designed to handle Wikipedia-level large corpora. Corpus2graph supports many language processing configurations, from word preprocessing to sentence analysis, and different ways of defining network edges and edge attributes. By using our node-edge-weight threelevel progressive calculation design, it can quickly build networks for multiple configurations.

\footnotetext{
${ }^{1} \mathrm{http}: / /$ textexture.com

${ }^{2}$ https://safetyapp.shinyapps.io/GoWvis/

3 available at https://github.com/zzcoolj/corpus2graph
} 
We are currently using it to experiment with injecting pre-computed word co-occurrence networks into word2vec word embedding computation.

\section{Efficient NLP-oriented graph generation}

Our tool builds a word co-occurrence network given a source corpus and a maximal distance $d_{\text {max }}$. It contains three major parts: word processing, sentence analysis and word pair analysis from an NLP point of view. They correspond to three different stages in network construction.

\subsection{Node level: word preprocessing}

The contents of large corpora such as the whole English Wikipedia are often stored in thousands of files, where each file may contain several Wikipedia articles. To process a corpus, we consider a file as the minimal processing unit. We go through all the files in a multiprocessing way: Files are equally distributed to all processors and each processor handles one file at a time.

To reduce space requirements, we encode each file by replacing words with numeric ids. Besides, to enable independent, parallel processing of each file, these numeric ids are local to each process, hence to each file. A local-id-encoded file and its corresponding local dictionary (word $\rightarrow$ local id) are created after this process. As this process focuses on words, our tool provides several word preprocessing options such as tokenizer selection, stemmer selection, removing numbers and removing punctuation marks. It also supports user-provided word preprocessing functions.

All these local-id-encoded files and corresponding local dictionaries are stored in a specific folder (dicts_and_encoded_texts). Once all source files are processed, a global dictionary (word $\rightarrow$ global id) is created by merging all local dictionaries. Note that at this point, files are still encoded with local word ids.

\subsection{Node co-occurrences: sentence analysis}

To prepare the construction of network edges, this step aims to enumerate word co-occurrences, taking into account word distance. Given two words $w_{1}^{i}$ and $w_{2}^{j}$ that co-occur within a sentence at positions $i$ and $j(i, j \in\{1 \ldots l\}$ where $l$ is the number of words in the sentence), we define their distance $d\left(w_{1}^{i}, w_{2}^{j}\right)=j-i$. For each input

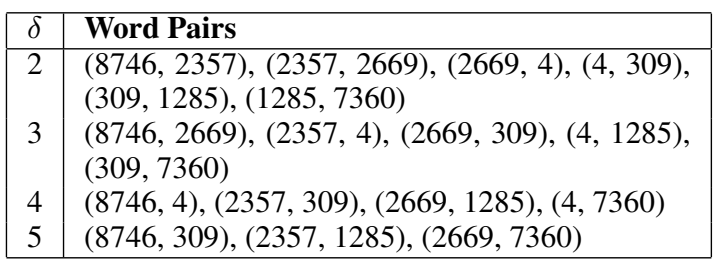

Table 1: Word pairs for different values of distance $\delta$ in sentence "8746 23572669430912857360 "

file, $d_{\max }$ output files will be created to enumerate co-occurrences: one for each distance $\delta \in$ $\left\{1, \ldots d_{\max }\right\}$. They are stored in the cooc folder.

To prepare the aggregation of individual statistics into global statistics (see Section 2.3), each process converts local word ids into global word ids through the combination of its local dictionary and of the global dictionary. Note that at this point the global dictionary must be loaded into RAM.

Then, a sentence analyzer goes through this file sentence by sentence to extract all word cooccurrences with distances $\delta \leq d_{\max }$. For instance, sentence "The NLP history started in the 1950s." may be encoded as "8746 235726694 309 1285 7360"; the sentence analyzer will extract word pairs from distance 1 to $d_{\max }$. The results for $d_{\max }=5$ are shown in Table 1.

User-customized sentence analyzer and distance computation are also supported so that more sophisticated definitions of word pair distance can be introduced. For instance, we plan to provide a syntactic distance: the sentence analyzer will build a parse tree for each sentence and compute word pair distance as their distance in the parse tree.

Besides, in this step, we also provide an option to count the number of occurrences of each word $o c c(w)$. Given that a large corpus like Wikipedia has a huge number of tokens, a global word count is convenient to enable the user to select words based on a frequency threshold before network generation. We return to this point in the next subsection.

\subsection{Edge attribute level: word pair analysis}

A word pair $\left(w_{1}, w_{2}\right)$ is represented by an edge linking two nodes in the word co-occurrence network. In this step, we enrich edge information with direction and weight by word pair analysis.

Let $\operatorname{cooc}\left(\delta, w_{1}, w_{2}\right)$ the number of cooccurrences of $w_{1}$ and $w_{2}$ with a distance of $\delta$ (Eq. 1). We define the weight $w\left(d_{\max }, w_{1}, w_{2}\right)$ of an edge $\left(w_{1}, w_{2}\right)$ as the total number of 
co-occurrences of $w_{1}$ and $w_{2}$ with distances $\delta \leq d_{\max }$ (Eq. 2).

$$
\begin{array}{r}
\operatorname{cooc}\left(\delta, w_{1}, w_{2}\right)=\left|\left\{\left(w_{1}^{i}, w_{2}^{j}\right) ; d\left(w_{1}^{i}, w_{2}^{j}\right)=\delta\right\}\right| \\
w\left(d_{\text {max }}, w_{1}, w_{2}\right)=\sum_{\delta \leq d_{\max }} \operatorname{cooc}\left(\delta, w_{1}, w_{2}\right)
\end{array}
$$

For efficiency we use an iterative algorithm (Eq. 3):

$w\left(d, w_{1}, w_{2}\right)= \begin{cases}\operatorname{cooc}\left(1, w_{1}, w_{2}\right), & \text { if } d=1 \\ \operatorname{cooc}\left(d, w_{1}, w_{2}\right)+ & \\ w\left(d-1, w_{1}, w_{2}\right), & \text { otherwise }\end{cases}$

We calculate the edge weight of different window sizes in a stepwise fashion by applying Eq. 3. For the initial calculation, we start by counting and merging all word pair files of distance 1 in the edges folder generated by step 2 to get a cooccurrence count file. This file contains information on all distinct word pair co-occurrence counts for distance 1 . We then follow the same principle to obtain a co-occurrence count file for distance 2 . We merge this result with the previous one to get word pair co-occurrence counts for window size 2 . We continue this way until distance $d_{\max }$.

If we wish to make further experiments with a larger distance, there is no need to recompute counts from the very beginning: we just need to pick up the word pair co-occurrences of the largest distance that we already calculated and start from there. All co-occurrence count files for the different distances are stored in the graph folder.

Defining the weight as the sum of cooccurrences of two words with different distances is just one of the most common ways used in graph-based natural language processing applications. We also support other (built-in and userdefined) definitions of the weight. For instance, when calculating the sum of co-occurrences, we can assign different weights to co-occurrences according to the word pair distance, to make the resulting edge weight more sensitive to the word pair distance information.

For a large corpus, we may not need all edges to generate the final network. Based on the word count information from Section 2.2, we may select those nodes whose total frequency is greater than or equal to min_count, or the most frequent vocab_size number of nodes, or apply both of these constraints, before building edges and computing their weights.

\section{Efficient graph processing}

\subsection{Matrix-type representations}

Although our tool works with graph libraries like igraph, NetworkX and graph-tool as a front end, we also provide our own version of graph processing class for efficiency reasons: Most graph libraries treat graph processing problems in a network way. Their algorithms are mainly based on network concepts such as node, edge, weight, degree. Sometimes, using these concepts directly in

(3)network algorithms is intuitive but not computationally efficient. As networks and matrices are interchangeable, our graph processing class uses matrix-type representations and tries to adapt network algorithms in a matrix calculation fashion, which boosts up the calculation speed.

In our matrix representation for graph information, nodes, edges and weights are stored in an adjacency matrix $A$ : a square matrix of dimension $|N| \times|N|$, where $N$ is the number of nodes in the graph. Each row of this matrix stands for a starting node, each column represents one ending node and each cell contains the weight of the edge from that starting node to the ending node.

Note that not all network algorithms are suitable for adapting into a matrix version. For this reason, our graph processing class does not aim to be a replacement of the graph libraries we mentioned before. It is just a supplement, which provides matrix-based calculation versions for some of the algorithms.

To give the reader an intuition about the difference between the common network-type representation and the matrix-type representation, the coming subsection uses the random walk algorithm as an example.

\subsection{Random walk}

Random walks (Aldous and Fill, 2002) are widely used in graph-based natural language processing tasks, for instance word-sense disambiguation (Moro et al., 2014) and text summarization (Erkan and Radev, 2004; Zhu et al., 2007). The core of the random walk related algorithms calculation is the transition matrix $P$.

In the random walk scenario, starting from an initial vertex $u$, we cross an edge attached to $u$ that leads to another vertex, say $v$ ( $v$ can be $u$ itself when there exists an edge that leads from $u$ to $u$, which we call a self-loop). Element $P_{u v}$ of the transition matrix $P$ represents the transition prob- 
ability $P(u, v)$ of the walk from vertex $u$ to vertex $v$ in one step. For a weighted directed network, $P(u, v)$ can be calculated as the ratio of the weight of the edge $(u, v)$ over the sum of the weights of the edges that start from vertex $u$.

NetworkX (version 2.0) provides a built-in method stochastic_graph to calculate the transition matrix $P$. For directed graphs, it starts by calculating the sum of the adjacent edge weights of each node in the graph and stores all the results in memory for future usage. Then it traverses every edge $(u, v)$, dividing its weight by the sum of the weights of the edges that start from $u$.

Based on the adjacency matrix $A$ introduced in Section 3.1, the transition probability $P(u, v)$ can be expressed as:

$$
P(u, v)=A_{u v} / \sum_{i=1}^{\left|A_{u}\right|} A_{u i}
$$

The transition matrix $P$ can be easily calculated in two steps: First, getting sums of all elements along each row and broadcasting the results against the input matrix to preserve the dimensions (keepdims is set to True); Second, performing element-wise division to get the ratios of each cell value to the sum of all its row's cell values. By using NumPy (Walt et al., 2011), the calculation is more efficient both in speed and memory. Besides, as the calculations are independent of each row, we can take advantage of multiprocessing to further enhance the computing speed.

\section{Experiments}

\subsection{Set-up}

In the first experiment, we generated a word cooccurrence network for a small corpus of 7416 tokens (one file of the English Wikipedia dump from April 2017) without using multiprocessing on a computer equipped with the Intel Core i7-6700HQ processor. Our tool serves as a front end to provide nodes and edges to the graph libraries NetworkX, igraph and graph-tool. In contrast, the baseline method processes the corpus sentence by sentence, extracting word pairs with a distance $\delta \leq d_{\max }$ and adding them to the graph as edges (or updating the weight of edges) through these libraries. All distinct tokens in this corpus are considered as nodes.

In the second experiment, we used our tool to extract nodes and edges for the generation of a word co-occurrence network on the entire English Wikipedia dump from April 2017 using 50 logical cores on a server with 4 Intel Xeon E54620 processors,$d_{\max }=5$, min_count $=5$ and vocab_size $=10000$.

In the last experiment, we compared the random walk transition matrix calculation speed on the word co-occurrence network built from the previous experiment result between our method and the built-in method of NetworkX (version 2.0) on a computer equipped with Intel Core i7-6700HQ processor.

\subsection{Results}

\begin{tabular}{lrrr}
\hline & NetworkX & igraph & graph-tool \\
\hline baseline & $\mathbf{4 . 8 8}$ & 8727.49 & 77.70 \\
corpus2graph & 15.90 & $\mathbf{1 4 . 4 7}$ & $\mathbf{1 4 . 3 1}$ \\
\hline
\end{tabular}

Table 2: Word network generation speed (seconds)

Table 2 shows that regardless of the library used to receive graph information generated by corpus2graph, it takes around 15 seconds from the small Wikipedia corpora to the final word cooccurrence network. And our method performs much better than the baseline method with igraph and graph-tool even without using multiprocessing. We found that in general loading all edges and nodes information at once is faster than loading edge and node information one by one and it takes approximately the same time for all graph libraries. As for NetworkX, the baseline method is faster. But as the corpora get larger, the baseline model uses more and more memory to store the continuously growing graph, and the processing time increases too.

For the second experiment, our tool took around 236 seconds for node processing (Section 2.1), 2501 seconds for node co-occurrence analysis (Section 2.2) and 8004 seconds for edge information enriching (Section 2.3). In total, it took less than 3 hours to obtain all the nodes and weighted edges for the subsequent network generation.

\begin{tabular}{lrr}
\hline Generation of: & network & transition matrix \\
\hline NetworkX & 447.71 & 2533.88 \\
corpus2graph & $\mathbf{1 1 6 . 1 5}$ & $\mathbf{1 . 0 6}$ \\
\hline
\end{tabular}

Table 3: Transition matrix calculation speed (seconds)

Table 3 shows the results of the third experiment. Loading network information into our graph processing class is faster than loading into the graph class of NetworkX. Moreover, our random 
walk transition matrix calculation method is 2390 times faster than the built-in method in NetworkX.

\section{Conclusion}

We presented in this paper an NLP-applicationoriented Python package that generates a word cooccurrence network from a large corpus. Experiments show that our tool can boost network generation and graph processing speed compared to baselines.

Possible extensions of this work would be to support more graph processing methods and to connect our tool to more existing graph libraries.

\section{References}

David Aldous and James Allen Fill. 2002. Reversible Markov chains and random walks on graphs. Unfinished monograph, recompiled 2014, available at http://www.stat.berkeley.edu/ aldous/RWG/book.html.

Ramon Ferrer i Cancho and Richard V. Solé. 2001. The small world of human language. Proceedings of the Royal Society of London B: Biological Sciences, 268(1482):2261-2265.

Gabor Csardi and Tamas Nepusz. 2006. The igraph software package for complex network research. InterJournal, Complex Systems:1695.

Günes Erkan and Dragomir R Radev. 2004. Lexrank: Graph-based lexical centrality as salience in text summarization. Journal of Artificial Intelligence Research, 22:457-479.

Olivier Ferret. 2004. Discovering word senses from a network of lexical cooccurrences. In Proceedings of the 20th International Conference on Computational Linguistics, COLING '04, Stroudsburg, PA, USA. Association for Computational Linguistics.

Aric A. Hagberg, Daniel A. Schult, and Pieter J. Swart. 2008. Exploring network structure, dynamics, and function using NetworkX. In Proceedings of the 7th Python in Science Conference (SciPy2008), pages 11-15, Pasadena, CA USA.

Rada Mihalcea and Paul Tarau. 2004. Textrank: Bringing order into text. In Proceedings of the 2004 conference on empirical methods in natural language processing.

Rada F. Mihalcea and Dragomir R. Radev. 2011. Graph-based Natural Language Processing and Information Retrieval, 1st edition. Cambridge University Press, New York, NY, USA.

Andrea Moro, Alessandro Raganato, and Roberto Navigli. 2014. Entity linking meets word sense disambiguation: a unified approach. TACL, 2:231-244.
Tiago P. Peixoto. 2014. The graph-tool python library. figshare.

François Rousseau and Michalis Vazirgiannis. 2013. Graph-of-word and tw-idf: New approach to ad hoc ir. In Proceedings of the $22 \mathrm{Nd}$ ACM International Conference on Information \& Knowledge Management, CIKM '13, pages 59-68, New York, NY, USA. ACM.

Stefan van der Walt, S. Chris Colbert, and Gael Varoquaux. 2011. The numpy array: A structure for efficient numerical computation. Computing in Science and Engg., 13(2):22-30.

Xiaojin Zhu, Andrew Goldberg, Jurgen Van Gael, and David Andrzejewski. 2007. Improving diversity in ranking using absorbing random walks. In Human Language Technologies 2007: The Conference of the North American Chapter of the Association for Computational Linguistics; Proceedings of the Main Conference, pages 97-104. 